Original Research Article

\title{
A double-blind randomized placebo-controlled study of low dose mirtazapine once daily in patients of major depressive disorders on escitalopram
}

\author{
Mallikarjuna Rao I. ${ }^{1}$, Usha Kiran Prayaga ${ }^{2 *}$, Dharma Rao Uppada ${ }^{3}$, Ramachandra Rao E. ${ }^{4}$, \\ B. L. Kudagi ${ }^{1}$
}

${ }^{1}$ Department of Pharmacology, NMC, Nellore, Andhra Pradesh, India

${ }^{2}$ Department of Pharmacology, RMC, Kakinada, Andhra

Pradesh, India

${ }^{3}$ Clinical pharmacologist,

VIMTA Labs Limited,

Hyderabad, Telangana, India

${ }^{4}$ Department of Psychiatry,

RMC, Kakinada, Andhra

Pradesh, India

Received: 07 March 2019

Revised: 23 March 2019

Accepted: 03 April 2019

*Correspondence to:

Dr. Usha Kiran Prayaga,

Email:

ushakiranprayaga@gmail.com

Copyright: (C) the author(s), publisher and licensee Medip Academy. This is an openaccess article distributed under the terms of the Creative Commons Attribution NonCommercial License, which permits unrestricted noncommercial use, distribution, and reproduction in any medium, provided the original work is properly cited.

\begin{abstract}
Background: The SSRIs being used as $1^{\text {st }}$ line therapy in treatment of depression have delayed therapeutic effect which makes the patient vulnerable to an increased risk of suicide and decreased adherence to the treatment and will prematurely discontinue the therapy. The present study was conducted to evaluate if low dose mirtazapine-escitalopram combination therapy has any add on benefit over monotherapy with escitalopram.

Methods: In a single-centered, comparative study involving patients with depression attending the out-patient after screening and exclusion, 60 eligible patients were randomly assigned to receive tablet mirtazapine $7.5 \mathrm{mg}$ plus tablet escitalopram $10 \mathrm{mg}$ intervention or tablet escitalopram $10 \mathrm{mg}$ plus placebo intervention in a double-blind 6-week treatment phase. The primary outcome measure was the change in the 17-item Hamilton Depression Rating Scale (HDRS) and Montgomery-Asberg Depression Rating Scale (MADRS) score from baseline. Participants were evaluated at baseline, $1^{\text {st }}, 2^{\text {nd }}, 4^{\text {th }}$ and $6^{\text {th }}$ week. Results were analyzed using Chi-Square test for adverse effects and independent t-test analysis for efficacy parameter.

Results: In the analysis of results at $6^{\text {th }}$ week the numbers of patients achieved remission in mirtazapine group are more with a p-value of 0.018 which is significant and the numbers of responders in mirtazapine group are also more which is statistically significant on chi-square test. There is no significant difference was observed between the two groups with reference to occurrence of adverse effect.

Conclusions: Adding low dose mirtazapine has an added benefit in terms of efficacy and getting remission early with more number of responders in the treatment of major depression.
\end{abstract}

Keywords: Montgomery-asberg depression rating scale, Major depressive disorder, 17-item hamilton depression rating scale

\section{INTRODUCTION}

Depression is most common psychiatric disorder with highest prevalence globally and is a chronic, potentially debilitating illness which has tempered the human psychological conditions since the beginning of recorded history. The lifetime prevalence of depression in various studies from Europe and the United States have estimated 
to be $5 \%$ to $12 \%$ in men and $9 \%$ to $26 \%$ in women. The studies conducted by Reddy VM et al, Nandi DN et al, had shown the prevalence of depression among Indian population is in a range of 7.9 to 8.9 per 1000 population. ${ }^{1,2}$ And it was twice in urban communities of India with a prevalence 19.4 per thousand. ${ }^{3}$ Acute depressive episodes are mostly attributed to a combination of environmental and genetic factors. Depression may also occur spontaneously among people who appear to lack any obvious genetic or environmental predisposition. ${ }^{4}$ The increased incidence of suicide rates among youth in India can be attributable to the increased burden of Major depressive disorder (MDD) among the young population. ${ }^{5}$ This explains the alarming state of MDD situation in India and need of a medical treatment which works better than existing.

The SSRIs being used as $1^{\text {st }}$ line therapy in treatment of depression have delayed therapeutic effect which makes the patient vulnerable to an increased risk of suicide and decreased adherence of the patient to the treatment and will prematurely discontinue the therapy. Mirtazapine is one of the antidepressants which has a noradrenergic and specific serotonergic antidepressant (NaSSA), which may be responsible for its rapid onset of action relatively to SSRIs. Mirtazapine acts by antagonizing the adrenergic $\alpha 2-$ autoreceptors and $\alpha 2$-heteroreceptors as well as by blocking 5-HT2 and 5-HT3 receptors. It enhances, therefore, the release of norepinephrine and 5-HT1Amediated serotonergic transmission. ${ }^{6}$ Mirtazapine has an added benefit in the treatment of major depression because of its unique mechanism compared to conventional SSRIs and SNRIs, in patients who require additional therapy and it had early onset of action in MDD patients. FDA had approved the drug with a dosage ranges $15 \mathrm{mg}, 30 \mathrm{mg}$ and $45 \mathrm{mg}$ in 1996 . $^{7}$ But there are studies which had shown that low dose of Mirtazapine i.e., $7.5 \mathrm{mg}$ of the drug when given as an add-on therapy to SSRIs had shown the beneficial effects. ${ }^{8,9}$ However, studies are limited regarding add-on therapy of low-dose mirtazapine and in these limited studies, mirtazapine as an add-on therapy majority have been done on patients who are on wide range of SSRIs therapy and are of open labeled.

The present study was conducted with a main objective, to evaluate if low dose mirtazapine-escitalopram combination therapy has any add-on benefit over monotherapy with escitalopram. This study was conducted on patients who are on escitalopram monotherapy without any other conventional anti-depressant drugs.

\section{METHODS}

Present study was a prospective, double-blinded, comparative, randomized, parallel group placebocontrolled study.

Patients with depression attending the out-patient Department of Psychiatry, Rangaraya medical college, Kakinada, Andhra Pradesh, India were taken.
Patients were enrolled starting from June 2014 and continued till August 2015. A total of 137 patients were enrolled.

\section{Inclusion criteria}

- $\quad$ Current Major Depressive Disorder (MDD) DSMIV diagnosis, confirmed by a structured diagnostic interview by a psychology therapist with 17 ItemHDRS score $\geq 18$,

- $\quad$ Age between 18 and 65 years,

- On escitalopram only for more than or equal to 4 weeks,

- No associated co-morbid conditions (e.g., hypertension, diabetes).

\section{Exclusion criteria}

Patients with psychosis or delusional disorders (Current or past), acute suicidal tendency, mental retardation, patients having a history of current substance abuse or dependence, a serious and unstable medical condition (e.g., pacemaker, porphyria) and history of epileptic seizures had been excluded from the study. Pregnant and lactating mothers were not eligible for the study.

\section{Intervention}

Tablet Mirtazapine 7.5 mg, brand name "Mirtaz 7.5 mg" (batch number EMN0606) and Tablet Escitalopram $10 \mathrm{mg}$, brand name "Nexito 10 mg" (batch number EMN0659) manufactured by Sun Pharmaceutical Industries Ltd., were given per oral every day at bedtime. The placebo tablet used in this study contains starch. Drugs are plastered with black sticker on both sides. The medications are labeled as A or B and dispersed by the nursing staff.

\section{Outcomes}

The primary outcome was the change in the HDRS- 17 and MADRS score from baseline to 6 weeks. Secondary outcomes included clinical response (defined as a $>50 \%$ reduction from the baseline HDRS-17 or MADRS score), and remission (defined as an HDRS-17 score $\leq 7$ or a MADRS score $\leq 10$ ) at week 6 .

\section{Procedure}

The patients who have accepted and completed the written informed consent were given the patient identification number (PID). They were screened for complete blood count, blood glucose levels, serum cholesterol levels, liver function tests, blood urea, serum creatinine, ECG and Thyroid Stimulating Hormone (TSH) testing was done at the baseline to rule out hypothyroidism.

Women of childbearing potential were given counselling for avoidance of pregnancy and urine pregnancy test (hCG) obtained at screening, at day 1 and at follow up. 
At the start of study, a self-administered questionnaire regarding socio-demographic factors (age and sex), duration of depressive symptoms and duration of treatment will be collected. Baseline 17-item Hamilton Depression Rating Scale (HDRS) score $\geq 18$ and Montgomery and Asberg depression rating scale (MADRS) score $\geq 18$ are noted in clinical research form by the investigator under supervision of psychiatry expert who is unaware of the trail group assignment (double blinded). In this study, participant/MDD patient is considered to be responded to treatment if there is a $50 \%$ or greater decrease in depression severity HDRS score from baseline to endpoint and remission is defined as a sub-threshold score on a depression scale, for example, a score of 7 or less on the 17-item Hamilton Depression Rating Scale is considered.

The sample size of 66 was calculated with an assumption of $30 \%$ difference will be observed between the two groups in terms of percentage of responders, alpha value of 0.05 and a power of 80 . But due practical and other constraints a total of sixty patients were given the investigational medication during the study period. The selected patients were assigned to two different intervention groups (group $\mathrm{A}, \mathrm{n}=30$ and group $\mathrm{B}, \mathrm{n}=30$ ) using Random numbers developed by online software. According to the interventional group that they belong the treatment is provided and is double-blinded so that the psychiatry expert and the patient receiving the treatment will not know which medication they are receiving. The randomization code and blinding are unmasked at the end of the study or if any serious ADR occurs.

Either of the medication (Either "Mirtazapine+Escitalopram" or "Escitalopram+Placebo") were given to the patient for 1 week depending on the group they belong to take once daily by oral route at bedtime. Follow-up of the participants were done on $1^{\text {st }}$, $2^{\text {nd }}, 4^{\text {th }}$ and $6^{\text {th }}$ week. And during the follow-up, HDRS and MADRS scores and adverse events were noted, and pills were provided for the treatment days until next scheduled follow up. Extra pills were also provided to the patient at day 0 and each follow up and are asked to bring remaining pills during each follow-up visit. Patients were instructed to note down any missed doses/broken pills/spilled pills from strip if any, on a patient diary which was provided at the start of the study. If the patient was illiterate, he/she was advised to take help of any literate in his household members or nearby. By means of pill count adherence to the regimens was determined and it was considered to be acceptable if less than $10 \%$ of the pills were returned.

The blinding was disclosed at the end of the study. The data was expressed as Mean \pm Standard error of mean (SE). The primary statistical analysis was intention to treat (ITT) analysis for all safety and efficacy variables with the last observation being carried forward (LOCF) for those patients who had at least 2 weeks data. Results were analyzed using Chi-Square test for adverse effects and independent t-test analysis for efficacy parameter. A P value of $<0.05$ was considered as statistically significant.
The study protocol was approved by the Dr. NTR University of Health Sciences, Vijayawada, Andhra Pradesh, India and the institutional ethics committee of the Rangaraya Medical College (IEC/RMC/13 MEP-001). The patients were explained and given complete adverse effects information of study drugs and were explained about the study details and a written informed consent was taken before enrolling them to the study. The study was done in accordance with ICH GCP guidelines.

\section{RESULTS}

In this study, a total of 137 patients were screened of which, 109 patients met inclusion criteria. Of the 109 patients, 90 patients agreed to give explained, written informed consent and remaining 16 refused to give consent and 3 patients refused to expose to placebo by randomization. Out of the 90 patients, 21 patients are excluded because of exclusion criteria and 9 patients left for other personal reasons leaving 60 patients receiving the allocated intervention. These 60 patients were randomly assigned in double-blind fashion to the treatment groups. At the time of analysis, the blinding was revealed. And it was "Tablet Mirtazapine 7.5 mg+Tablet Escitalopram $10 \mathrm{mg}$ ” intervention provided to group A patients and group B patients had received "Tablet Escitalopram 10 mg+placebo.” Baseline demographic characteristics are depicted in Table 1.

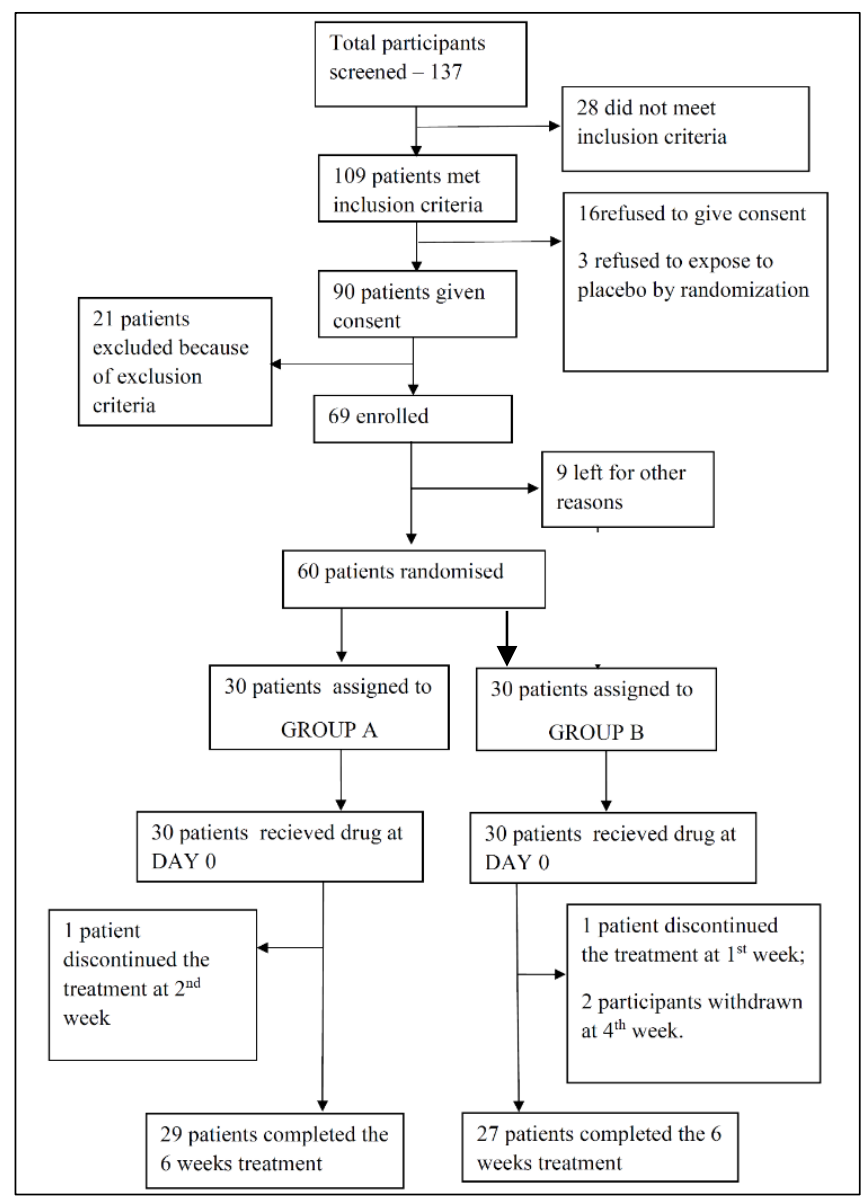

Figure 1: Participants study plan. 
A total of 56 (29 with group A intervention and 27 with group B intervention) patients had completed the 6 weeks follow-up assessment. Participants study plan shown in Figure 1. Both completers and dropouts are characteristically similar in baseline symptom severity measures. As intention to treat analysis was done for the analysis complete data of 29 patients in each group was available.

Table 1: Baseline characteristics of study population.

\begin{tabular}{|c|c|c|c|c|c|}
\hline \multicolumn{2}{|l|}{ Characteristic } & \multicolumn{2}{|c|}{ Mirtazapine group $(n=30)$} & \multicolumn{2}{|c|}{ Placebo group $(\mathrm{n}=30)$} \\
\hline \multirow{4}{*}{ Age categorized } & 18-30 years & 10 & & 10 & \\
\hline & $31-40$ years & 10 & & 11 & \\
\hline & $41-50$ years & 6 & & 4 & \\
\hline & 51-65 years & 4 & & 5 & \\
\hline \multirow{2}{*}{ Gender } & Male & 12 & & 13 & \\
\hline & Female & 18 & & 17 & \\
\hline & & Mean \pm SD & SE & Mean \pm SD & SE \\
\hline \multicolumn{2}{|c|}{ Duration of illeness in weeks } & $6.2 \pm 1.1$ & 0.21 & $6.5 \pm 1.1$ & 0.20 \\
\hline \multicolumn{2}{|l|}{ HDRS } & $23.2 \pm 3.1$ & 0.56 & $23.8 \pm 2.7$ & 0.50 \\
\hline \multicolumn{2}{|l|}{ MADRS } & $27.8 \pm 2.9$ & 0.53 & $29.1 \pm 2.7$ & 0.50 \\
\hline \multicolumn{2}{|l|}{ Blood glucose } & $81.7 \pm 11.9$ & 2.17 & $84.2 \pm 11.8$ & 2.15 \\
\hline \multicolumn{2}{|l|}{ Serum cholesterol } & $135.9 \pm 21.9$ & 3.87 & $146.9 \pm 18.3$ & 3.35 \\
\hline \multicolumn{2}{|l|}{ Serum bilirubin } & $0.8 \pm 0.22$ & 0.04 & $0.697 \pm 0.15$ & 0.03 \\
\hline \multicolumn{2}{|c|}{ Alkaline phosphatase } & $76.5 \pm 17.5$ & 3.19 & $59.2 \pm 11.2$ & 2.04 \\
\hline \multicolumn{2}{|c|}{ Aspartate aminotransferase } & $26.6 \pm 6.1$ & 1.12 & $18.7 \pm 3.6$ & 0.65 \\
\hline \multicolumn{2}{|c|}{ Alanine transaminase } & $26.2 \pm 5.5$ & 1.00 & $25.1 \pm 6.4$ & 1.17 \\
\hline
\end{tabular}

HDRS: Hamilton depression rating scale, MADRS: Montgomery and Asberg depression rating scale, SD: Standard deviation, SE: Standard error of mean.

Table 2: Efficacy parameters within the group efficacy of intervention.

\begin{tabular}{|c|c|c|c|}
\hline Mirtazapine group & Mean difference \pm SD & SE & P value \\
\hline Difference in HDRS between baseline and week 4 & $12.0 \pm 3.1$ & 0.58 & $<0.001^{*}$ \\
\hline Difference in HDRS between baseline and week 6 & $14.9 \pm 3.1$ & 0.58 & $<0.001^{*}$ \\
\hline \multicolumn{4}{|l|}{ Placebo group } \\
\hline Difference in HDRS between baseline and week 4 & $9.0 \pm 2.1$ & 0.40 & $<0.001 *$ \\
\hline Difference in HDRS between baseline and week 6 & $12.2 \pm 2.9$ & 0.54 & $<0.001 *$ \\
\hline
\end{tabular}

HDRS: Hamilton depression rating scale, MADRS: Montgomery and Asberg depression rating scale, SD: Standard deviation, SE:

Standard error of mean.

\section{Efficacy}

The mean of HDRS score in mirtazapine group at $4^{\text {th }}$ and $6^{\text {th }}$ week were $11.4 \pm 4.6$ and $8.4 \pm 3.8$ respectively, whereas the same were $14.8 \pm 3.4$ and $11.6 \pm 3.4$ in placebo group. The difference observed between the groups was statistically significant on independent-t-test. The mean of MADRS score in mirtazapine group at $4^{\text {th }}$ week and $6^{\text {th }}$ week were $15.1 \pm 2.4$ and $11.9 \pm 2.3$ respectively, whereas the same were $18.2 \pm 3.1$ and $15.2 \pm 2.8$ in placebo group. The same differences were found to be statistically significant on independent t-test. Based on the reduction in HDRS scores at the end of study from baseline, the efficacy of intervention was evaluated. In mirtazapine group, the mean reduction of HDRS score from baseline till $6^{\text {th }}$ week was $14.9 \pm 3.1$ which was statistically significant on pairedt-test with a $\mathrm{P}$ value of $<0.001$ and in placebo group, the mean reduction of HDRS score from baseline till $6^{\text {th }}$ week was $12.2 \pm 2.9$, which was also statistically significant on paired-t-test with a $\mathrm{P}$ value of $<0.001$. Within the group efficacy parameters of intervention are depicted in Table 2.

\section{Responders}

At $4^{\text {th }}$ week the numbers of responders in Mirtazapine group are 16 (55.2\%) and in the placebo group the responders are $5(17.2 \%)$ which is statistically significant on chi-square test with a p-value of 0.003 and the odds ratio, 5.91 (95\% confidence interval: $1.8-19.8)$. At $6^{\text {th }}$ week the numbers of responders in mirtazapine group are 24 (82.8\%) and in the placebo group, the responders are 15 (51.7\%) which is statistically significant on chi-square test (p-0.012, odds ratio=4.48, 95\% CI:1.3-14.9). 


\section{Remission rate}

At $4^{\text {th }}$ week the numbers of patients achieved remission in mirtazapine group are $9(31.0 \%)$ and in the placebo group remission achieved by $2(17.2 \%)$ patients with a p-value of 0.019 which is significant and the odds ratio with $95 \%$ confidence interval is $6.07(1.2-31.2)$. At $6^{\text {th }}$ week the number of patients achieved remission in mirtazapine group are 18 (62.1\%) and in the placebo group remission achieved by 9 (31.0\%) patients with a p-value of 0.018 which is significant (OR-3.64,95\% CI:1.2-10.7). The mean HDRS scores at day 0 and on follow-up of two groups are shown in Figure 2.

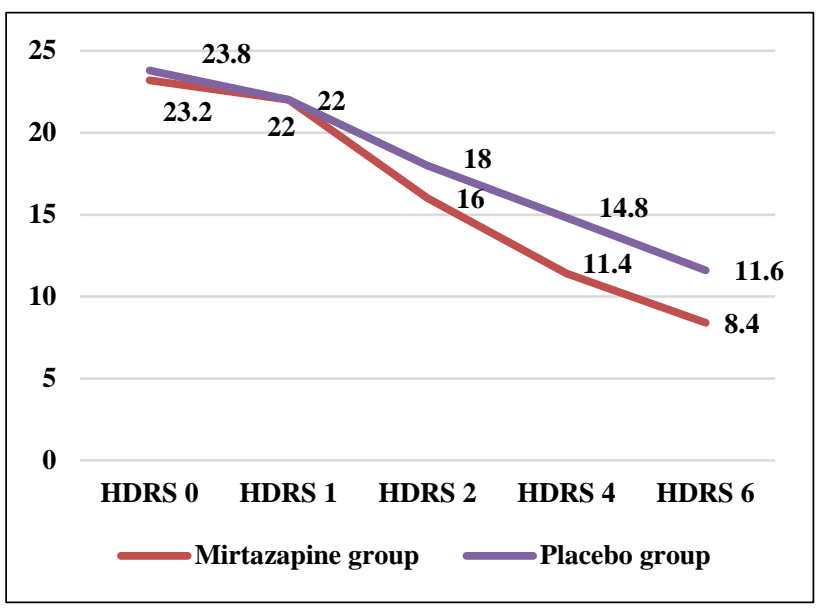

Figure 2: Line diagram showing the mean HDRS scores at day $\mathbf{0}$ and on follow-up of two groups.

\section{Safety profile}

The adverse events (AE) observed among the patient population in mirtazapine group were fatigue (17.2\%), dry mouth (34.5\%), nausea or vomiting (10.3\%), insomnia (10.3\%), headache (17.2\%), serum cholesterol (10.3\%), increased appetite $(13.8 \%)$, significant weight gain at $6^{\text {th }}$ week (17.2\%). The adverse effects shown by patients in placebo group were fatigue (13.8\%), dry mouth (17.2\%), nausea or vomiting (10.3\%), insomnia (17.2\%), headache (31.0\%), serum cholesterol (3.4\%), increased appetite (3.4\%), significant weight gain at $6^{\text {th }}$ week $(3.4 \%)$. There is no significant difference was observed between the two groups with reference to occurrence of adverse effect. There was no serious adverse event reported.

Significant weight gain was defined by gain of at least $7 \%$ of body weight from baseline, 5 patients in mirtazapine group and 1 patient in placebo group showed significant weight gain at $6^{\text {th }}$ week. In mirtazapine group, 13 patients have reported that there is a betterment of sleep whereas in placebo group the same is reported by 3 patients, which had been statistically significant on chi-square test. Patients in mirtazapine intervention group had 7 times more chance to have better sleep patterns compared to placebo group (OR7.04,95\% CI:1.73-28.6).

\section{DISCUSSION}

In the present study, there was clear-cut difference observed in both MADRS and HDRS scales which favoured low-dose mirtazapine $7.5 \mathrm{mg}$ add on treatment in comparison with escitalopram and placebo in both group and between-group analyses. Earlier studies have shown that a greater number of responders at an earlier time period when mirtazapine was used as add on therapy. ${ }^{8,9}$ The same was observed in this study even though there was significant difference found between placebo and mirtazapine group.

In a previous trial by Matreja PS et al, the HDRS mean difference between baseline and $6^{\text {th }}$ week was $12.83 \pm 0.41$, which is less than present study observation. Although difference is observed with this study the efficacy in terms of reduction in the depression scale scores are corresponding to the results of the present study, where statistically significant difference between mirtazapine and placebo started to be evident after four weeks of treatment in both the studies. This can be attributed to the single drug escitalopram as the main treatment used in present study in contrast to a range of SSRIs used in the study mentioned above. $^{10}$

In current study, by $4^{\text {th }}$ week $31.0 \%$ participants in mirtazapine group and $6.9 \%$ in placebo group attained remission which is similar to previous studies conducted by Carpenter LL et al, where the remission at $4^{\text {th }}$ week is observed in $45.5 \%$ in mirtazapine combination group and in placebo group it was $13.3 \%{ }^{11}$ The difference can be due to higher dose of mirtazapine $(15-30 \mathrm{mg})$ in the earlier mentioned study and the HDRS-17 score of $>12$ was the inclusion criteria in contrast to HDRS-17 score of $\geq 18$ in this study. ${ }^{11}$

In Blier $\mathrm{P}$ et al, the number of patients who achieved a response to treatment at $6^{\text {th }}$ week were fluoxetine monotherapy, 54\%, mirtazapine plus fluoxetine, 68\%, mirtazapine plus bupropion, 65\%, mirtazapine plus venlafaxine, $73 \%$ and found that there was no statistical difference between the groups. ${ }^{12}$ But in this study, the response rates between the two groups were significant statistically $(\mathrm{P}<0.01)$ and the same in mirtazapine group is $82.8 \%$ and in placebo group $51.7 \%$. This difference can be attributed to the sample size in this study is bigger than earlier ones and, in this study, mirtazapine used as lowdose which might decrease the dropout rate and achieved the better response. In contrast to Blier P et al, study, author continued the escitalopram treatment among patients, which was given at least for 4 weeks prior to the study and added to that mirtazapine and placebo were given during the intervention.

In the current study, both the groups were well tolerated, and the adverse events observed were mild and transient. It is found that $34.5 \%$ of patients had a complaint of dry mouth in mirtazapine group and the same was $17.2 \%$ in escitalopram group. This finding is similar to that of 
previous trials. ${ }^{13,14}$ Other Adverse Events (AEs) found in mirtazapine intervention group of present study were weight gain at $6^{\text {th }}$ week and fatigue.

Even though somnolence is one of the main problems with mirtazapine, in this study patients reported that instead of somnolence there was a betterment of sleep. ${ }^{11}$ Betterment of sleep is observed in $44.8 \%$ of the patients in mirtazapine group, compared to only $10.3 \%$ of patients in placebo group. Similar to Winokur A et al, study where there was reduced sleep latency had been attributed to mirtazapine. This difference was highly significant statistically. This can be attributed to the low dose of mirtazapine which author used in the study. As the dose of mirtazapine increases the beneficial effects of the drug wanes as observed and stated by the previous reviews and studies. ${ }^{13-}$ 16

In this study, author found there is an increase in weight at $6^{\text {th }}$ week in $17.2 \%$ of the patients in mirtazapine group in contrast to $3.4 \%$ patients in placebo group. However, there was no statistically significant difference found.

In this study, there is transient rise in serum cholesterol in majority but within normal limit except in 3 members there is rise in serum cholesterol more than normal but no statistical significant association has found which is similar to studies of Nicholas LM et al, on healthy subjects for 4 weeks and Laimer $\mathrm{M}$ et al, on depressed and healthy subjects for 6 weeks. ${ }^{17,18}$

Combining Medications to Enhance Depression Outcomes (CO-MED) study (2011) had shown that extended-release venlafaxine plus mirtazapine was associated with a greater side effect burden at 12 weeks and 7 months than escitalopram plus placebo and a higher number of worsening adverse events (AEs) than escitalopram plus placebo at 7 months. ${ }^{19}$ However, in the current study as the low dose mirtazapine $7.5 \mathrm{mg}$ was added to escitalopram 10 mg for 6 weeks duration, is the differentiating factor from the CO-MED study and the reason for less frequency in the AEs. In the earlier mentioned study, the dose of venlafaxine ranges from $37.5 \mathrm{mg}$ to $300 \mathrm{mg}$ and mirtazapine dose ranges from $15 \mathrm{mg}$ to $45 \mathrm{mg}$ can be implicated to high number of adverse events. ${ }^{19}$ Unlike the studies with SSRIs in MDD which had shown that, the insomnia is the major residual symptom in this study it was not the major concern and only three patients had complained of insomnia. ${ }^{9,20}$

Previous studies have shown that stepped care model as the best treatment modality in MDD patient, where the default management is that patients start with an evidence-based treatment of low intensity (treatments that require less time from a professional) as a first step. Progress is monitored systematically and those patients who do not respond adequately will step up to a subsequent treatment of higher intensity. This will consume more time from the professional expert to confirm the well suitable treatment specific to the patient and patient himself/herself need spend more time on multiple visits. ${ }^{21}$ In this context, as found in the current study, that the combination of low dose mirtazapine of $7.5 \mathrm{mg}$ with escitalopram as a first-line treatment is having an advantage to escitalopram monotherapy in terms of remission rate. Improvement in symptoms due to mirtazapine add-on treatment have been reported in several RCTs, which is similar to current study. The strengths of the present study are that patient population was presented with initially moderate to high scores, which makes it possible to extrapolate its results to patients with difficult-to-treat depression. Only study which was conducted with strict inclusion criteria and is double-blinded in India. An RCT phase, which makes its results reliable from the viewpoint of the evidence-based medicine. The drop-out rate was very low due to continuous mobile reminders and easy access to the clinic for patients which also improves the reliability of the results.

Limitation of this study are that patients were treated for only 6 weeks, which is insufficient time to know the full remission rates. The dose of escitalopram monotherapy may have been low. The sample size was small. The patients in this study may not be entirely representative of depressed patients who are typically seen in practice having concurrent general medical and psychiatric comorbidity.

\section{CONCLUSION}

Low-dose mirtazapine $7.5 \mathrm{mg}$ has an added benefit in terms of efficacy and getting remission early in the treatment of major depression because of its noradrenergic and specific serotonergic antidepressant mechanism compared to conventional SSRIs/SNRIs monotherapy and it had early onset of action in MDD patients. This study had shown that a greater number of responders at an earlier time period when mirtazapine was used as add on therapy. The results of current study support the concept of using escitalopram with mirtazapine $7.5 \mathrm{mg}$., as a first-line treatment or at second step in the treatment of MDD patients of moderate to severe depression. Further efforts to evaluate the safety, efficacy, and place for low-dose mirtazapine medication with $7.5 \mathrm{mg}$ dose as an added therapy to escitalopram are called for in even large sample size of patients.

\section{ACKNOWLEDGEMENTS}

Authors would like to thank Dr. R. Mahalakshmi (M.S. Principal of Rangaraya Medical College, Andhra Pradesh, India) and patients, who participated, for their support during study.

\section{Funding: No funding sources Conflict of interest: None declared}

Ethical approval: The study was approved by the Institutional Ethics Committee of the Rangaraya Medical College, Andhra Pradesh, India (IEC/RMC/13 MEP-001) 


\section{REFERENCES}

1. Reddy VM, Chandrashekar CR. Prevalence of mental and behavioural disorders in India: a meta-analysis. Ind J Psychiatry. 1998;40(2):149.

2. Nandi DN, Banerjee G, Mukherjee SP, Ghosh A, Nandi PS, Nandi S. Psychiatric morbidity of a rural indian community: changes over a 20-year interval. Brit J Psychiatry. 2000;176(4):351-6.

3. Sethi BB, Prakash R. Depression in Industrial population. Ind J Psychiatry. 1979;21(4):359.

4. Koda-Kimble MA, Alldredge BK. Applied therapeutics : the clinical use of drugs. 10th ed. Philadelphia: Wolters Kluwer/Lippincott Williams and Wilkins; 2013: 2519.

5. Reddy MS. Depression: the disorder and the burden. Ind J Psychol Med. 2010;32(1):1.

6. Anttila SA, Leinonen EV. A review of the pharmacological and clinical profile of mirtazapine. CNS Drug Rev. 2001;7(3):249-64.

7. Drugs@FDA: FDA Approved Drug ProductsREMERON, Available at: https://www.accessdata.fda.gov/scripts/cder/ob/result s_product.cfm?Appl_Type=NandAppl_No=020415.

Accessed 30 September 2018.

8. Papakostas GI, Homberger CH, Fava M. A metaanalysis of clinical trials comparing mirtazapine with selective serotonin reuptake inhibitors for the treatment of major depressive disorder. J Psychopharmacol. 2008;22(8):843-8.

9. Nierenberg AA, Keefe BR, Leslie VC, Alpert JE, Pava JA, Worthington III JJ, et al. Residual symptoms in depressed patients who respond acutely to fluoxetine. J Clin Psychiatry. 1999;60(4):221-5.

10. Matreja PS, Badyal DK, Deswal RS, Sharma A. Efficacy and safety of add on low-dose mirtazapine in depression. Ind J Pharmacol. 2012;44(2):173.

11. Carpenter LL, Yasmin S, Price LH. A double-blind, placebo-controlled study of antidepressant augementation with mirtazapine. Biol Psychiatry. 2002;51(2):183-8.

12. Blier P, Ward HE, Tremblay P, Laberge L, Hébert C, Bergeron R. Combination of antidepressant medications from treatment initiation for major depressive disorder: a double-blind randomized study. Am J Psychiatry. 2009;167(3):281-8.
13. Croom KF, Perry CM, Plosker GL. Mirtazapine. CNS Drugs. 2009;23(5):427-52.

14. Stimmel GL, Dopheide JA, Stahl SM. Mirtazapine: an antidepressant with noradrenergic and specific serotonergic effects. Pharmacotherapy J Human Pharmacol Drug Therapy. 1997;17(1):10-21.

15. Winokur A, Sateia MJ, Hayes JB, Bayles-Dazet W, MacDonald MM, Gary KA. Acute effects of mirtazapine on sleep continuity and sleep architecture in depressed patients: a pilot study. Biol Psychiatry. 2000;48(1):75-8.

16. Thase ME, Nierenberg AA, Vrijland P, Oers HJ, Schutte AJ, Simmons JH. Remission with mirtazapine and selective serotonin reuptake inhibitors: a metaanalysis of individual patient data from 15 controlled trials of acute phase treatment of major depression. Int Clin Psychopharmacol. 2010;25(4):189-98.

17. Laimer M, Kramer-Reinstadler K, Rauchenzauner M, Lechner-Schoner T, Strauss R, Engl J, Deisenhammer EA, et al. Effect of mirtazapine treatment on body composition and metabolism. J Clin Psychiatry. 2006;67(3):421-4.

18. Nicholas LM, Ford AL, Esposito SM, Ekstrom RD, Golden RN. The effects of mirtazapine on plasma lipid profiles in healthy subjects. J Clin Psychiatry. 2003;64(8):883-9.

19. Rush AJ, Trivedi MH, Stewart JW, Nierenberg AA, Fava M, Kurian BT, et al. Combining medications to enhance depression outcomes (CO-MED): acute and long-term outcomes of a single-blind randomized study. Am J Psychiatry. 2011;168(7):689-701.

20. Ogrodniczuk JS, Piper WE, Joyce AS. Residual symptoms in depressed patients who successfully respond to short-term psychotherapy. J Affective Dis. 2004;82(3):469-73.

21. Straten A, Hill J, Richards DA, Cuijpers P. Stepped care treatment delivery for depression: a systematic review and meta-analysis. Psychol Med. 2015;45(2):231-46.

Cite this article as: Rao MI, Prayaga UK, Uppada DR, Rao RE, Kudagi BL. A double-blind randomized placebo-controlled study of low dose mirtazapine once daily in patients of major depressive disorders on escitalopram. Int J Basic Clin Pharmacol 2019;8:1067-73. 